Membres, - $\mathbf{I}^{\circ}$ Section Lyonnaise : MM. Boutan, Administrateur-Délégué de la Compagnie du Gaz de Lyon. E.-F. Côte, Professeur à l'Ecole Centrale Lyonnaise ; GodiNET, Président de la Société Générale de Force et Lumière ; Henrard, Administraleur-Délégué de la Société des Forces Motrices du Rhône; Praton, Administrateur de la Chambre Syndicale des Forces Hydrauliques, Président de la Société d'Economie Politique de Lyon.

$2^{\circ}$ Section Grenobloise : MM. Barbillion, Directeur de l'Institut Polytechnique de l'Université de Grenoble ; Barưt, Administrateur de la Chambre Syndicale des Forces Hydrauliques, Administrateur-Délégué de la Société Electrochimique du Giffre; Aimé Boucharer, Administrateur de la Chambre Syndicale des Forces Hydratiques, Administrateur de la Société Générale de Forces Motrices el d’Eclairage de la Ville de Grenoble; Léprne, Secrétaire du Conseil de la Chambre Syndicale des Forces Hydrauliques, Administrateur-Délégué de la Société Hydro-Electrique de Fure et Morge et de Vizille; Vienromme, Vice-Président de la Chambre Syndicalc des Forces Hydrauliques, Président de la. Société Electrométallurgique Francaise.

Trésorier: - M. Gharpenay, Trésorier de la Chambre Synidicale des Forces Hydrauliques, Administrateur-Délégué de la Société des Forces Motrices du Haut-Grésivaudan.

Secrétaire. - X...

Nous tiendrons nos lecteurs au courant de l'organisation de ce Congrès auquel ils adhéreront en grand nombre, nous l'esperons.

\section{LES CONSÉQUENCES JURIDIQUES DE L'ABANDON DU LIT PAR UNE RIVIÈRE non navigable, ni flottable}

"Il y a des morts qu'il faut qu'on tue " I Ce vieil adage a été souvent employé pour caractériser le souvenir un peu encombrant de certaines personnalités, dont on parle encore, alors même qu'elles n'existent plus. Mais; si étrange que cela paraisse, il faut encore l'appliquer à l'ancienne rédaction de l'article 563 du Code civil, qui, avant d'être modifié formellement par la loi du 8 avril I 898 , prévoyait l'hypothèse d'un cours d'eau quelconque abandonnant son lit, et réglementait la situation des propriétaires dépossédés par la nouvelle direction des caux. L'imagination féconde des barreurs trouve le moyen de l'invoquer encore, sous prétexte que les droits que leur donnait autrefois l'ancien texte ont survécu à sa disparition, sauf, biẹn entendu, à se réclamer des principes contenus dans la loi nouvelle, s'ils y trouvent un intérêt.

Pauvre article 563 ! II aura eu une douloureuse histoire, puisquc, critiqué dans sa premièrc forme, il n'est point encore arrivé à satisfaire tout le monde. Nous voudrions, dans les lignes qui ront suivre, en préciser la portée en indiquant les grands principes de la propriété, dont est susceptible le lit d'une rivière, et quelles sont aujourd'hui les conséquences juridiques d'un changement de lit.

\section{I}

Lce texte de l'ancien arlicle 563 était ainsi conçu : « Si un "fleuve ou unc rivière, navigable, flotlable on non, se forme " un nouveau cours, en abandonnant son ancien lit, les " propriétaires des fonds nouvellement occupés, prennent " à titre d'indemnité l'ancien lit abandonné, chacun dảns "la proportion du terrain qui lui a été enlevé ".
Ainsi qu'on le voit, par les premiers mots de l'article, le Code ne faisait aucune différence entre le lit abandonné par' les rivières publiques, c'est-à-dire les rivières navigables ou flottables, et le lit des rivières ni navigables ni flottables, c'est-à-dire de colles que l'on appelle quelquefois des rivières privées.

Au moment de la rédaction du Gode civil, il y eut sur ce texle une très vive discussion devant le. Conseil d'Etat. Plusieurs personnalités marquantes de cette asscmblée réclamaient l'application si simple du droit romain et voulaient attribuer aux propriétaires riverains la pleine propriété de la surface laisséé libre par te changement de direction survenu dans le cours de la rivière. Maleville soutint alors, peut-être avec une certaine témérité, que, même dans les provinces de droit écril, c'est-à-dire dans celles où le droit romain était encore journellement appliqué, les tribunaux avaient abandonné la vieille loi latine, et, par des motifs d'équité, consacré une jurisprudence nouvelle, par laquelle l'attribution du lit à titre de compensation, était faile aux propriétaires des parcelles nouvellement recouvertes par los eaux.

Ge fut cetle demière thèse qui prévalut; el depuis le Code civil, plus en théorie qu'en pratique peut-être, on vit cette compensation se faire par l'attribution du terrain recouvert au propriétaire du terrain découvert.

Cette disposition s'explique par les idées recues en I804. A cette date, toute rivière était considérée plutôt comme un ennemi, à cause de ses débordements; des érosions constantes et des dangers de son voisinage. D'où cette conclusion qu'il fallait donner aux propriétaires dépossédés par le nouveau passage des eaux une compensation sous forme d'un terrain disponible, tandis qu'au contraire le riverain qui perdait sa riveraineté élait considéré comme relativement heureux, puisqu'il perdait une proximité gènante. Comme il aurait élé bon, à cetle époque, avec de pareilles théories de faire l'acquisition des droits de riveraineté pour établir des chutes d'eau dans l'avenir.

Plus l’on s'écarta de l'année 180 /4, plus les riverainctés prirent de l'importance. Si bien, que l'article 563 fut commenté parlout d'une façon fort désavantageuse. Je ne parle pas, bien entendu, des professeurs et des étudiants, plus nombreux qu'on le croit, qui, n'ayant jamais vu que des grandes villes et des rivières navigables, s'imaginent que toul cours d'eau, si petit qu'il soit, cst toujours entouré de quais ou au moins de digues. IIs ont affecté de traiter par l'absurde un article ne visant d'après eux qu'unę hypòthèse absolument chimérique, les ruisseaux n'abandonnant jamais leur lit...

Mais je ne m'occupe que des praticiens de l'hydraulique. Aucun d'entre cux ne dira quc le cas prévu est une puro utopie ; car tous ceux qui onl été témoins de débordements formidables, comme celui du Charmaix en rgo6, n'ignorent rien de la cause de ces changements de lit ; ils savent que la précipitation de l'eau est si violente qu'elle enlève sur son passage tous les obstacles, se charge de boues et do sable, devient une masse liquide d'une considérable densité, et fait flotter à sa surface des rochers énormes, qui ressemblent à un morceau de fer flottant sur une cuve de mercure ; dès que la pluie s'arrête, cette masse, perdant de sa vitesse, laisse tomber au fond du lit les blocs qu'elle entraînait ; ceux-ci, formant barrage, détournent l'eau à droite et à gauche si bien que l'ancien lit, sans cau, mais couvert de pierres, est complètement délaissé et se garnit rapidement de broussailles et de végétations de hasard.

On comprend sans peine - et sur ce point les critiques 
peuvent librement se faire jour sans craindre d'exagérer quelles complications devait produire l'attribution du sol nolivellement découvert au propriétaire du sol nouvellement recouvert. Dans la plupart des cas cette idée de justice qui avait présidé à la rédaction de la loi et lui servait d'excuse ne pouvait être satisfaite. Quelle consolation le propriétaire d'un champ bicn cultivé, vraiment producteur, peut-il trouver dans le fait de recevoir un terrain chargé de pierres, de graviers et de détritus de toutes sortes ? Si l'on veut même supposer que les valeurs fussent identiques, on devra reconnaître au moins que, souvent pour accéder à cette terre dite de compensation, le moindre chemin praticable faisait défaut. Enfin, pour répartir entre les nouveaux propriétaires les surfaces acquises tout en maintenant une proportion rigoureuse, calculée d'après les surfaces enlevées, il fallait se livrer à des arpentages, à des calculs, à des lotissements qui pratiquement étaient impossibles.

Aussi, lorsque l'on se décida à refaire une loi nouvelle sur le régime des eaux, l'ancienne rédaction de l'article 563 fut condamnéc à mort; cela se passa en 1898 .

Tout d'abord, on renonça au texte unique englobant à la fois les rivières navigables el les rivières non navigables. Le Code Civil fut réservé aux rixières de la première catégorie. Nous ne voulons point nous en occuper aujourd'hui. Disons seulement que d'après le nouveau texte, si un fleuve abandonne son ancien lit, les propriétaires riverains peuvent acquérir la surface laissée libre, à charge d'en payer à l'Etat qui est propriétaire le prix fixé par des experts nommés par le tribunal civil.

S'ils ne font point usage, dans un délai convenu, de ce droit de préemption, l'aliénation de l'ancien lit est faite, conformément aux règles de l'aliénation du domaine de l'Etat. Et le prix de la vente est distribué à titre d'indemnité aux propriétaires des fonds submergés par le nouveau cours.

Quant à la situation des propriétaires submergés par les cours d'eau non navigables ni flottables, elle fut facilement réglée, grâce à la théorie nouvellement admise par la loi, d'après laquelle tout riverain d'un cours d'eau de cette nature est propriétaire du lit jusqu'à la ligne 'médiane de la rivière : propriété certaine qui lui a souvent donné la possibilité de s'opposer à ce que le riverain d'en face, au moyen d'un barrage, dépasse cette frontière établie par la loi. Lorsque les eaux abandonnent ce lit, celui-ci ne change pas de maître, et reste à son premier propriétaire qui peut alors le cultiver comme un terrain ordinaire, on un mot, en jouir comme un sol dont il retrouve la pleine jouissance. Et, d'autre part, le propriétaire dont le terrain, par suite d'un caprice des eaux, est recouvert, ne perd pas une parcelle de sa propriété, pưisque même par dessous ces caux, cette propriété subsiste encore. Il voit seulement disparaître la culture du sol nouvellement submergé et doit supporter ce fait du hasard. Mais la loi se devait à elle-même de prévoir que chacun des propriétaires peut être mécontent de son sort. Le premier, qui a vu fuir la rivière, dans une autre direction, va déplorer sa riveraineté perdue, tandis que l'autre, qui a vu les eaux se précipiter sur un sol arable, so considérera comme très peu compensé par la riveraineti qu'il acquiert. Aussi la loi leur permet à chacun d'eux de prendre dans l'année qui suit le changement de lit les mesures nécessaires pour rétablir l'ancien cours des eaux.

Enfin, si l'abandon du lit n'est pas naturel, mais provient de travaux légalement ordonnés, les propriétaires des terrains occupés ont droit à une indemnité, non pas pour perte de propriété mais pour perte de jouissance.
Telle est la signification des articles 4,5 et 6 de la loi du 8 avril $\mathrm{r} 898$.

Article 4. - Lorsque le lit d'un cours d'eau est abandonné, soit naturellement, soit par suite de travaux légalement exécutés, chaque riverain en reprend la libre disposition suivant les limites déterminées par l'article précédent.

Article 5. - Lorsqu'un cours d'eau non navigable ni flottable abandonne naturellement son lit les propriétaires des fonds sur lesquels le nouveau lit s'établit, sont tenus de souffrir le passage des eaux sans indemnité, mais ils peuvent dans l'année qui suit le changement de lit prendre les mesures nécessaires pour rétablir l'ancien cours des eaux.

Les propriétaires riverains du lit abandonné jouissent de la même faculté et peuvent, dans l'année, poursuivre l'exć. cution des travaux nécessaires au rétablissement du cours primitif.

Article 6. - Lorsque par suite de travaux légalement ordonnés il y a licu d'élargir le lit ou d'en ouvrir un nouveau, les propriétaires des terrains occupés ont droit à ume indem. nilé à titre de servitude de passagc...

\section{II}

On peut se demander, avec un certain étonnement, comment des dispositions si claires, si catégoriques, de la loi de $\mathrm{r} 898$, ont pu donner lieu et peuvent même donner licu de nos jours à des contestations séricuses.

ll est aisé cependant de le comprendre, en sc meltant en présence de l'hypothèse suivante.

Supposons un changement de lit survenu à une époque qui, bien qu'antérieure à la loi de 1898 , ne soit pas encore séparée de nous par un intervalle trentenaire. Admettons, par exemple, qu'cn 1897 le propriétaire d'une parcelle sitú́ sur la rive gauche de la rivière ait vu une partic de son terrain submergée par les eaux : par le fait de ce brusque mouvement, une parcelle sur la rive droite a surgi, en quelque sorte, étant mise à découvert par le changement de lit; et il est arrivé, ce qui se produit toujours : les voisins ont utilisé cette parcelle devenue libre ; et, tout doucement, par la force même des choses, en l'absence de toute réclamation, l'ont incorporée à leur fonds. Enfin, pour prendre une espèce vécue, supposons que, sur cette partie ainsi incorporée, ils aient établi une usine.

Un beau jour, le propriétaire de la rive gauche recouverte par les eaux écrira au propriétairc do la rive droite; "En vertu de l'article 563 du Code Civil, je suis devenu " propriétaire de la parcelle découverte en 1897 , et en droit " cette propriété m'a été attribuéc du jour où mon terrain "a été recouvert par les eaux. Or, vous connaissez le grand " principe que la propriélé ne se perd jamais à moins qu'um " autre l'ait prescrite à son profit. Je réclame aujourd'hui "le sol dont j'étais devenu propriétaire avant la loi de r 808 " et que vous n'avez pas pu prescrire, faute de temps. Ne " mo dites point que cette loi a un effet rétroactif. Sí l'évé" nement s'était produit postérieurement à cette loi, je se" rais le premier à reconnaître que nos rapports respectifs " devraient être réglés par elle. Mais elle n'a pas pu me dé" posséder d'un droit que j'avais acquis avant son appari" tion et l'on ne peut m'appliquer un texte qui n'est va"lable que pour l'avenir".

Que devra répondre le propriétaire qui, depuis seize ans, a pris l'habitude de considérer la terre incorporée comme sa propriété définitive ? On m'accordera bien volontiers que ce n'est point une discussion d'école, mais un problème vrajment juridique et important.... 
Aussi, à celui qui devra répondre el défendre son usine contre les prétentions du riverain d'en face, il faut avant tout donner un sage conseil : celui d'être très clair, de ne point embrouiller la question, et pour cela de ne pas feuilleter des recueils de jurisprudence qui ne feraient que l'effrayer. Il y verrait, en effet, toute une série de discussions sur le caractère interprétatif ou non de la loi de $18{ }_{9} 8$, sur des divergences de vues qui se sont produites entre la Chambre des Requêtes et la Chambre civile de la Cour de Cassation sur les questions épineuses qui n'ont rien à voir dans la solution du problème : heureusement pour lui, cette solution est plus simple. La voici :

Toute loi, d'après un grand principe, qui ne trompera jamais personne, est applicable au jour de sa promulgation, mêmes aux situations ou rapports juridiques formés avant son apparition.

C'est l'évidence pure et simple, et l'on se demande comment on interpréterait les lois nouvelles si, à chaque instant, sous le prétexte que les rapports juridiques entre deux personnes ont commencé sous l'empire de la loi ancienne, c'est celle-ci qu'il faudrait appliquer au lieu de la loi nouvelle. C'est ce que disent en termes excellents MM. Aubry ct Rau, tome I, $\S 30$, $5^{\circ}$ édition, pages $98-99$ : " En principe, " toute loi nouvelle s'applique même aux situations ou rap" ports juridiques élablis ou formés dès avant sa promul" gation. Ce principe est une conséquence de la souveraineté " de la loi el de la prédominance de l'intérêt public sur les " intérêts privés. Toutefois, ce principe doit faire place à la " règle contraire, dans les cas où son application serait de " nature à entraîner la lésion de droits que des particuliers " auraient individuellement acquis en ce qui concerne leur " état ou leur patrimoine $n .(1)$.

En somme, la loi de 1898 doit subir le sort de toutes les lois : elle est soumise à la grande distinclion qui existe entre les droits acquis qui appartiennent pour toujours à ceux qui les ont mis matériellement en œuvre, ou les ont déjà fait examiner par un tribunal, avant la modification de la loi, et les droits qui auraient pu seulement être revendiqués; ces derniers ne peuvent plus être demandés suivant l'ancienne législation, à partir du jour où celle-ci a été définitivement modifiée; voilà pourquoi, dans le cas qui nous occupe, le propriétaire menacé dans l'établissement de son usine par le riverain dépossédé autrefois, devra répondre : " Du moment que vous n'avez rien demandé avant la loi " de 1898 , et que vous n'avez pas appréhendé le terrain, " vous êtes forclos du droit de réclamer cette compensation "que l'ancienne loi vous attribuait".

Et en répondant ainsi on se conformerait intégralement à un arrêt de la Cour de Cassation en date du i I décembre IgoI, qui a confirmé ce principe ${ }^{2}{ }^{2}$.

Cet arrêt a été rendu dans une instance assez bizarre entre deux propriétaires dont l'un, s'appuyant sur la loi nouvelle, voulait établir une séparation entre son fonds et le fonds d'en face, en se basant sur l'attribution en pleine propriété, que la loi venait de consacrer pour chacun d'eux sur la partie du lit déterminée par la ligne médiane. Le propriétaire assigné en bornage répondait avec raison que la loi r 898 n'a pas eu pour effet de permettre cetle limitation, car la ligne médiane, qui fait la séparation, n'a pas une fixité suffisamment rigoureuse : mais, dans sa réponse à l'assignation, il avait essayé de lancer une fin de non recevoir,

(1) Cette référence est donnée dans le Dalloz, sous l'arrêt de Joybert contre Laval-Noiset en date du 2 décembre 1901. Dal. 1902. L. 353.

(2) Voir la référence ci-dessus. basée sur ce que l'instance, née le 28 décembre 1897 , ne pouvait même pas être invoquée dans l'affaire. Sur ce point, la Cour a décidé que le Tribunal avait bien fait de considérer comme applicable à l'instance la loi nouvelle qui avait fait son apparition à un moment où cette affaire était déjà lancée devant les tribunaux. C'est une bonne leçon pour la question que nous étudions : le propriétaire menacé pourra donc répondre au réclamant : " Alors même que vous " m'auricz demandé la compensation résultant de l'art. 563, " avant l'application de la loi de $r 898$, vous auriez dû aban" donner votre instance, si pendant son cours la promulga" tion de la loi nouvelle était intervenue : a fortiori, vous vous n'avez plus le droit de demander l'application de cet article, ni d'invoquer cette prétendue propriété immédiate qu'il consacrait, puisque votre instance vient au monde dix-sept ans après la promulgation de la loi ".

Enfin, il y aurait encore un argument à faire valoír qui est le suivant : même sous l'empire de l'ancien article 563 , le raisonnement du demandeur aurait été inexact: on ne saurait trop répéler que, comme le fait si bien observer Picard (Tome I, page 327), si l'ancien lit était autrefois attribuć aux riverains dépossédés, ce n'était point à titre de propriété spontanée, mais à titre d'indemnité : "Dans plusieurs de ces arrêts, dit le savant auteur, la Cour Suprême a insistó sur ce caractère de l'attribution édictée " par le Code Civil. Elle a notamment décidé que l'acqué" reur d'un fonds lraversé par une rivière ne devenait pas "de plein droit proprićtaire de l'ancien lit et ne pouvait le "revendiquer comme une dépendance naturelle de ce "fonds. "-V. Cass. Req., ar décembre $187^{4}$. Dal. $7^{6}$, I, 43 I : Affaire Scrratrice c. Communc de Rancurel (1).

On peut alors se demander quelle procédure devait suivre le riverain dépossédé pour se faire donner la part à laquelle il avait droit : puisqu'il s'agissait de recevoir un terrain à titre d'indemnité, cela ne pouvait avoir licu qu'en la réclamant au propriétaire du lit devenu libre par le nouveau cours des eaux.

Or, ce propriétaire, à l'époque où l'article 563 était en vigueur, ne pouvait être que l'Etal : en effet, ou il s'agissait d'une rivière navigable ou flottable dont il fallait, par hypothèse, occuper l'ancien lit, et, par conséquent, l'Etat en était propriétairc en vertu de l'article 538 du Code Civil; ou bien il s'agissait d'une rivière non navigable ni flottable, el, comme à celle époque la propriété du lit des cours d'eau de cette nature n'était point encore fixée, l'Etat en devenait propriétaire, parce qu'il est, d'une façon générale, en vertu de l'article 539 du Code Civil, propriétaire de tous les biens qui n'ont point de maître.

(1) Cet arrêt de la Cour de Cassation a rejeté le pourvoi contre un arrèt de la Cour d'appel de Grenoble en se servant des termes reproduits ci-clessus par PIGird.

Comme la Cour de Grenoble avait au mois de mai 1875 conflrmé par adoplion de motifs le jugement du Tribunal de St-Marcellin, du 16 mai 1872, nous avons eu la curiosité de faire venir co dernier document, et nous y avons vu ce qui suit: en 1851 le ruisseau de Doulouche s'était formé un nouveau lit sur le sol de Glénat-Tardiva dont Serratrice est devenu propriétaire aux termes d'un procès-verbal d'adjudication du 22 septembre 1867: or, dans ce procès-verbal d'adjudication n'état pas compris l'ancien lit du Doulouche; en rait il etait constant que Serratrice n'était pas en possession de la parcelle revendiquée: Serratrice avait donc plaidé qu'en droit son auteur avait dû devenir propriétaire presque automatiquement, depuis le jour où le ruisseau s'était déplacé, de la partje laissée libre à titre de compensation : c'est à cela que' la Cour Suprême répond par ces mots: "Attendu que ]'ancien lit abana donné par une rivière qui s'est formé un nouveau cours ne constitue " point un accessoire ou une dépendance naturélle des fonds nouvellement " occupés et que ce n'est pas en cette qualité, mais à titre d'indemnité " que les propriétaires de ces fonds sont appelés à prendre l"ancien lit "abandonné chacun dans la proportion qui lui a détenlevée. 
Il aurail donc fallu, sous l'empire de l'article 563, que le riverain, dépossćdé par la nouvelle direction des eaux, fît une instance contre le Préfet représentant l'Elat pour obtenir soit une attribution en nature, à titre d'indemnité de dépossession, soit en argent, à la suite d'une licitation opérée, au cas où le partage aurait été absolument impossible.

\section{III}

Mais celte application de la loi de 1898 est tellement inléressante que nous ne pouvons nous empeccher, par une transition toute naturelle, d'étudier une question, voisine de la nôtre, bien qu'elle ne soit pas relative à un changement de lit : loutefois elle y touche de très près.

Ne peut-on pas, dans certains cas, bien que cette loi proclame d'une façon absolue que le riverain est propriétaire du lit qui touche sa rive, trouver encore des riverains qui, acluellement, sont déboutés de leur demande en reconnaissance de celte propriété, même postérieurement à la loi de $x 898 ?$

Il faut répondre affirmativement, et même ajouter que cela n'implique aucune contradiction avce ce qui vient d'être dit : au contraire, on aurait déjà pu le déduire de la citation que nous avons faite de MM. Aubry et Rau, qui déclarent la loi de 1898 applicable à toules les instances nées postérieurement à son apparition, mais à la condition qu'il ne soit pas porté échec, par ce moyen, à un droit acquis.

Et, puisque nous avons eu soin de définir le droit acquis comme étant celui qui a été mis matériellement en œuvre, ou déjà examiné par un tribunal, nous n'avons qu'à chercher dans la jurisprudence quels sont les exemples de droits mis en ceuvre, ou de droits au moins virtuellement présentés ì la justice.

Un arrêt de lạ Cóur d'Aix (1), en date du 7 avril rgo2, impitoyablement cassé par la Cour de Cassation en date du I I janvier r $905\left({ }^{2}\right)$, nous fournit le meilleur exemple. Un sieur Roussel avait vu créer par l'Administration, en $188_{7}$, une scrvitude de halage sur un terrain lui appartenant, au bord de la rivière " le Large " qui, probablement en suite de travaux effectués par l'Administration, était devenue une rivière publique.

Entre ce chemin de halage et la rivière, il restait un pelit terrain que l'on appelle dans le stylc administratif un horsligne, el que l'Administration est tenue d'acquérir si sa surface présente les deux particularités suivantes : être inférieure au quart de la surface totale de la propriété de l'exproprié, et encore être inférieure à to ares. Le sieur Rousset, pour établir la première condition, c'est-à-dire prouver que le hors-ligne n'alteignait pas le quart de la surface totale, était obligé de comprendre dans ladite surface, la portion du lit qui, d'après la loi acluclle, aurait élé sûrement sa propriété : et, en $x 887$, ayant sur cette loi une avance de onze ans, il présenta son argumentation au jury d'expropriation. Celui-ci régla l'affaire, comme il le fait d'habitude, en attribuant une indemnité hypothétique au sieur Rousset, c'est-à-dire en lui disant que s'il faisait établir le bien-fondé actuel de sa prétention par le Tribunal civil, il aurait droit à cefte indemnité, tandis qu'au contraire, s'il échouait dans sa prétention, il ne lui serait rien accordé, l'Administration pouvant alors se refuscr à acquérir.

Par quel mystère, ou par suite de quelles lenteurs, le sieur Rousset ne présenta-t-il au Tribunal. Civil la thèse qui hii ćlait chère, que plus de 13 ans après l'année 1887 ? Nous

(1) Dalloz, 1903. I. 151

(2) Dalloz, 1905. 1. 163. l'ignorons absolument, mais loujours est-il que la loi de 1898 était rendue quand le Tribunal Civil de Forcalquier eut à staluer, à la dale du 23 mai rgor : il s'empressa, des lors, d'appliquer celle loi, d'en prononcer l'cffet rétroactif, disant qu'elle comblait une lacune de la loi ancienne, et que, le sieur Rousset avait toujours censé être propriélaire du lit de la rivière. La Cour d'Aix suivit le Tribunal dans son argumentation.

Mais la Cour Suprême a cassé l'arrêt de la Cour d'Aix par le raisonnement suivant: A quelle époque le droit de Rousset et le droit contraire de l'Administration se sont-ils affirmés devant un Tribunal ? En d'autres termes, à quelle époque l'Administration a-t-elle déclaré qu'clle trouvait dans la législation existante un droil acquis lui permettant de considérer le sieur Roussel comme n'élant pas propriétaire du lit de la rivière le Large ? ll faut évidemment répondre que c'est la date a laquelle, par une discussion souverainement conslatée dans une décision passée en force de chose jugće, les deux parties litigantes, c'est-à-dire le sieur Roussel el lo Préfet des Basses-Alpes, onl plaidé leurs droits respectils devant le jury. C'est par conséquent à l'année r887 que le Tribunal et la Cour d'Aix auraicnt dû se reporter par la pensée pour définir les droits dont ils élaient saisis par suile du renvoi prononcé par le magistrat directeur du jury.

Donc, le sieur Rousset aurait dû êlre déboulé, puisqu’a ce moment l'ancienne législation, au moins telle que l'interprélait la jurisprudence de la Cour de Cassation, avait toujours tenu pour certain que le lil de la rivière élail une res nullius, comme l'est encore aujourd'hui l'eau qui court au-dessus ; tel est le molif de la cassation de l'arrêt de la Cour d'Aix.

La Cour suprême a d'ailleurs confirmé à nouveau cello jurisprudence dans un arrêt en dale du ${ }^{\text {er }}$ mars 1909 dans l'alfaire commune de Thézan-les-Bézicrs c. un sicur Arnaud. On y lit celte phrase très précise : "Altendu qu'au moment "où le jury avail slatué, la loi du 8 avril 1898 n'élait pas " encore promulguće ; que la Cour de Montpellier ne pou" vait done se fonder exclusivement sur les dispositions do "l'article 3 de celte loi pour décider, à l'encontre de la com" mune qu'Arnaud était, à l'époque de l'expropriation, pro"priétairc du point litigieux ". ( ${ }^{1}$ ).

Ces deux arrêts ont encore une aulre porléc pratique : c'est de faire considérer comme complètement abandonnée la thèse que quelrues Cours d'Appel, et même la Cour de Cassalion, dans un arrêt Duplan c. Ott, du I6 février rgol, avait adoptée, savoir la Joi de 1898 aurait seulement un caractère interprétalif et, par conséquent, serait toujours applicable à toutes les discussions même nées antérieurement à son apparition, si bien que, sans distinguer cntre l'hypothèse de droits acquis, el de droits à revendiquer, il faudrait

(1) On a lait aussi un assez grand état d'un arret te la Cour d'Appol de Tyon ( $\Lambda$ f:. Malleval, c. Berchoux du 5 août 1904) et corlfrmé par la Cour Suprême par arrêt du 26 février 1906 (Dalloz 1908, I. 502), la Cour de Lyon paraît admetitre que si l'Administration a flxé le long d'une rivière un plan dalignoment, datant de 1820 , au dela doquel les riverains n'aumient jamais ou lo droit de construire, il y a dans ce plan d'aliguement, officinl obligatoire pour lous une sorle de titre ou de droit acquis, d'après loquel lo riverain d'en face pout s'opposer a ce gu'un riverain construise sur la partie da lit, hien que la loi de 1808 ait fait lattribution que l'on sitit.

Mais ce qui paraît nvoir ote la cause détorminante, on fait, de la dósisión intervenue, costla constatation par la Cour de Lyon que les construetions établies par le sieur Malleval genajunt l'coulement des eaux, au prejudice du sieur Berchonx, et pat conséquent violaient l'article 10 de la loi du. 8 avril 1898: ainsi concu : Le proprietaire riverain d'un cours "d'eau non navigable ni flottable ne peut exéculer des travaux au"dessus de ce cours doau ou le joignant quat la condition de no pas "préjudicier à l'écoulement et de no causer aucun dommage aux "proprieles voisines." 
toujours en faire l'application sans autre forme de procès : comme on le voil, par les arrếs précilés, la Cour admet aujourd'hui qu'en réalité la loi de isgs a innové, qu'elle a donné aux riverains une propriélé qu’ils ignoraient complètement avant elle, et que la jurisprudence antéricure leur refusail. A partir du jour où la loi a été promulguée, les riverains peuvent revendiquer la propriété du lit, ils sont done soumis à celle loi, aclivement el passivement, pouvant in réclamer le bénélice, à moins que, en le réclamant, ils ne lèsent des droils mis en ceuvre ou déjà cnvisagés par un Iribunal, sous l'empire de la législation ancienne.

Au surplus, la lọi de 1898 semblail elle-même l'indiquer par les mots "sauf titre ou prescription contraires", qu'elle avail el soin d'insérer dans son texte : "Si les deux rives "apparlicnnent à des proprétaires différenls, chacun d'cux " a la propriété de la moitić du lit, suivant unc ligne que " l'on suppose tracée au milicu du cours d'eau, sauf. tilr'e "ou prescription contraire".

Du reste, il ne faut pas trop exagérer l'imporlance de celle querclle d'école qui s'est produile au sujet de la loi interprélative ou non : on sait que d'une façon générale, par larticle 2 du Code Civil, la loi ne dispose que pour l'avenir cl n'a pas d'effel rélroactif : mais on admet généralement que cette disposition ne vise pas les lois qui ne feraient que compléter les lois antérieures, el éclaircir des points que celles-ci auraient laissés obscurs. Toulefois, en ce qui concerne Ja loi de 1898 , on ne peul pas dire qu'elle ait fait un éclaircissement d'une situalion indécise, puisqu'en réalité elle a altribué une propriélé vraiment nouvelle, aux riverains des cours d'cau non navigables ni flotlables : et, cûl-elle même ćlé inlerprélalive, que, puisqu'elle devait respecler, d'après son texle, les droils acquis par titres ou par prescription, l'offet n'en aurait guère été modifié.

Disons-le même franchement : on peut très bien supposer que les doux principaux arrêts qui ont proclamé la thèse du caractère interprétatif de la loi auraient pu se dispenser de formuler celte théorie, et qu'ils n'en auraient pas moins été conduits à juger, élant donné les fails, d'une façon absolument identique.

C'est d'ailleurs ce que nous allons examiner.

Dans le premier arrêt, celui de la Cour d'Appel de Grenoble (Delhomme c. Daru, du 3 I octobre $x \delta_{99}$, rapporté dans Dalloz rgoo, deuxième partie, page 350 ), les faits se présenlaient de la façon suivante : sur une parcelle siluée en face des rives dans le lit de la rivière, Delhomme avait placé une canalisation d'cau et un petit pont : les sieurs Daru, qui voulaient continuer à emprunter le lit de la rivière devant Ja rive de Delhomme, pour aller d'un fonds leur appartenant à un autre de leurs fonds, ne se gênèrent pas autrement et supprimèrent simplement la passerelle, si bien qu'à la date du I 2 avril ${ }_{1} 8_{97}$ Delhomme dut agir contre eux : il demanda au Tribunal de le considérer comme propriétaire du lit du ruisseau sur lequel s'exerçail ce passage et assigna les consorts Daru, leur faisant au surplus défense de praliquer tout passage au droit de son fonds.

Sur ces entrefailes, la loi de $x 898$ intervint, et la Cour de Grenoble déclara que celle loi était applicable à la prétention de Delhomme, parce que c'était une loi d'ordre interprélatif ; ct pour prouver ce dernier point elle fit une longue disserlation : il aurait ćtć aussi simple et autrement inallafuable de dire, comme l'a dit la Chambre Civile de la Cour Suprême, dans l'affaire rappclée au début de cet arlicle (Joybert e. Iaval-Noiset), que la loi de i $\delta_{9} \delta$ devait s'appliquer à lous les procòs, même ceux pendants au moment de sa promulgation; qu'en conséquence Delhomme avail paríaitenenl raison de revendiquer la propriélé de la parlie du lit siluce en face de sa rive, el qu'il n'aurail pu être paralysé dans son droit que si les sieurs Daru avaienl pul invoquer an lilre ou une possession contrajes, ou évidemment un obslacle juridique, lel que l'enclave par cxemple. Or, précisément, la Cour de Grenoble avait remarqué en fait que Daru, pour se crécr un droil de passagc, ne pouvail alléguer et n'alléguait aucun titre, ni aucun argument lui permetlant d'invocuer un droil acquis relalivement au passage. On voil que la discussion sur le caractère interprétalif ou non de la loi élait absolument inulile.

Il en cst rigourcusement de même pour l'arrêt de la Cour de Cassation rendu dans l'affaire Ott c. Duplan, à la dale du 16 févier rgo4 (Dalloz rgo4, I, 263). La Cour de Nîmes, pour se dire en droit de juger la réclamation de la dame 014 d'après la loi de $\mathbf{1} 898$, avait cru nécessaire de dire que cello loi n'avail qu'un caraclère interprétalif, et que par conséquent elle était applicable à une situation juridique née avant sa promulgation : la Cour de Cassation la suivit dans cette discussion et en a approuvé la conclusion.

Il aurait élé infiniment plus simple de constater que la loi doit être appliquée dans tout jugement qui est rendu au moment où sa promulgation est une chose faite, et d'aulro part les adversaires de la dame Ott n'avaient aucun titre ni aucun droit acquis pour refuser à celte dame le droit de sc dire proprićlaire d'une partie du lit de la Volane : en effet, dans un acte de partage de 1834 il était rcconnu que l'autcur de la dame Ott était propriétaire d'un terrain riverain do cette rivière, et c'est dans cet élat que ce terrain s'était perpétué dans la famille : le 8 avril $\mathrm{r} 898$, la dame Ott voit arriver une loi qui étend sa propriété jusqu'au milieu d'u lit do la Volane : il n'y avail qu'à appliquer cetle loi, puisque la Cour avait elle-même constalć que les adversaires de la dame Ott, simplement en fail, de leur propre autorité privée, sans aucun titre ni aucune prescription, s'étaient installés sur celte partie de la rivière : le déguerpissement est de règle à l'encontre de tous ccux qui occupent sans titre ni prescription, la propriélé d'autrui, celte propriété ne fût-elle que l'humble lit d'une rivière non navigable ni flottable...

Paul Bougault, Amorat à la Cour d'Appel de Lyon.

\section{LA BAUXITE ET LE FOUR ÉLECTRIQUE}

Nous donnons ci-après la conférence faite en mai dernier à la Société Internationale des Electriciens par notre collaborateur M. Fuusir, professeur à la Faculté des Sciences de Grenoble, conférence annoncée dans notre numéro de septembre dernier à propos du "Probleme de la fixation industriclle de l'azole".

Le four électrique permet de faire subir à une même malière première, la bauxite, des transformations diverses, dont l'élude présente quelque intérèt, au point de vue industriel comme au point de vue scientifique.

La bauxite est un minerai de composition fort variable, constitué essentiellement par de l'alumine hydratéc impure ; les impurelés normales sont l'oxyde forrique, la silice et, l'oxyde de titane. Fn France, où les giscments do bauxile sont particulièrement nombreux et abondants, on distingue deux qualités principales de minerai: Ja bauxile rouge, richo en oxyde de fer et pauvre en silice; la bauxitc. blanche, pauvre on oxyde de fer et riche en silice. Cette distinclion n'a rien d'absolu. On connaît par cxemple des bauxites blanches qui sont à la fois paurres en oxyde de fer et en silice; 